\title{
Direct Chiral Separation of $N$-Fluorenylmethoxycarbonyl $\alpha$-Amino Acids by HPLC for Determination of Enantiomeric Purity
}

\author{
Yin Hua Li, Chae-Sun Baek, Byung Wook Jo, ${ }^{\dagger}$ and Wonjae Lee* \\ College of Pharmacy, Chosun University, Gwangju 501-759, Korea. EE-mail: wlee@chosun.ac.kr \\ ${ }^{\dagger}$ Department of Chemical Engineering, Chosun University, Gwangju 501-759, Korea \\ Received March 28, 2005
}

Key Words : Enantiomer separation, Chiral stationary phase, $N$-FMOC $\alpha$-amino acids

\begin{abstract}
$N$-Protected $\alpha$-amino acids have been widely used as important chiral building blocks in the fields of pharmaceutical chemistry and biochemistry. The fluorenylmethoxycarbonyl (FMOC) group is one of the most commonly used protecting groups for $\alpha$-amino acids and provides the advantages of high sensitivity in fluorescence detection. ${ }^{1-3}$ Especially, the FMOC protecting group is attractive, because it is frequently used for amino protecting groups in solid phase synthesis and combinatorial chemistry. ${ }^{4,5}$ Owing to the importance of optical purity of $N$-FMOC $\alpha$-amino acids, convenient and accurate methods to determine the enantiopurity of these compounds have been required and developed. Several methods for the liquid chromatographic separation of the enantiomers of $N$-FMOC $\alpha$-amino acids have been reported using CSPs derived from cyclodextrin, macrocyclic antibiotics and cellulose derivatives as well as small molecules derived brush-type CSPs. ${ }^{6-13}$ Although the enantioseparation of some FMOC secondary amino acids has been performed on 1-naphthylethyl carbamoylated $\beta$ cyclodextrin bonded CSP with good resolution, ${ }^{6}$ most FMOC $\alpha$-amino acids enantiomers have not been resolved on $\beta$ - and $\gamma$-cyclodextrin bonded CSPs. ${ }^{7}$ It has been reported that nine $N$-FMOC $\alpha$-amino acids enantiomers were separated on macrocyclic antibiotics derived CSP. ${ }^{8,9}$ Brushtype CSPs derived from $\alpha$-amino acids and cinchona derivatives including Whelk-O CSP have been employed to resolve some FMOC $\alpha$-amino acids enantiomers. ${ }^{10-12}$ Also, the enantioresolution of two FMOC $\alpha$-amino acid type enantiomers has been reported on cellulosic-type CSP under reversed-phase conditions. ${ }^{13}$ For resolution of $N$-FMOC $\alpha$ amino acids, however, polysaccharide-derived CSPs have not been applied under normal phase conditions and the enantiomer separation of a series of these analytes has not been well investigated. ${ }^{14}$ In this study, the liquid chromatographic resolution of $N$-FMOC protected $\alpha$-amino acids for determination of their enantiomeric purity was performed on polysaccharide-derived CSPs, Chiralcel OD and Chiralpak AD under normal phase conditions. ${ }^{15,16}$

Table 1 and Table 2 show liquid chromatographic results for the separation of the enantiomers of $N$-FMOC $\alpha$-amino acids on Chiralcel OD and Chiralpak AD. Chiralcel OD and/ or Chiralpak AD afford fairly good enantioselectivities for the resolution of $N$-protected FMOC $\alpha$-amino acids under normal phase conditions. Chiralcel OD affords comparable
\end{abstract}

Table 1. Enantiomer Separation of $N$-FMOC Protected $\alpha$-Amino Acids on Chiralcel OD

\begin{tabular}{clrccc}
\hline Entry & \multicolumn{1}{c}{ Analyte } & $\mathrm{k}^{\prime}{ }^{a}{ }^{a}$ & \multicolumn{1}{c}{$\alpha^{b}$} & $\mathrm{Rs}^{c}$ & Retained $^{d}$ \\
\hline 1 & Alanine & 5.47 & 1.79 & 5.14 & $\mathrm{~L}$ \\
2 & Aminobutyric acid & 4.94 & 1.37 & 2.84 & $\mathrm{~L}$ \\
3 & Aminocaprylic acid & 4.27 & 1.39 & 2.71 & \\
4 & Asparagine & & \\
5 & Aspartic acid & 8.81 & 1.60 & 1.84 & $\mathrm{~L}$ \\
6 & Glutamic acid & 9.28 & 1.32 & 1.83 & $\mathrm{~L}$ \\
7 & Glutamine ${ }^{f}$ & 13.74 & 1.17 & 0.85 & $\mathrm{D}$ \\
8 & Isoleucine & 4.10 & 1.50 & 3.51 & $\mathrm{D}$ \\
9 & Leucine & 4.91 & 1.25 & 1.95 & $\mathrm{D}$ \\
10 & Methionine & 7.41 & 1.13 & 1.07 & $\mathrm{~L}$ \\
11 & Norleucine & 4.48 & 1.15 & 1.21 & $\mathrm{~L}$ \\
12 & Norvaline & 4.77 & 1.08 & 0.63 & $\mathrm{~L}$ \\
13 & Phenylalanine & 8.09 & 1.10 & 0.67 & $\mathrm{~L}$ \\
14 & Phenylglycine & 7.54 & 1.69 & 3.50 & $\mathrm{D}$ \\
15 & Serine $^{e}$ & 2.55 & 2.55 & 5.09 & $\mathrm{~L}$ \\
16 & Threonine $_{17}$ & 6.94 & 1.49 & 3.03 & $\mathrm{~L}$ \\
18 & Tyrosine $^{e}$ & Valine $^{19.60}$ & 1.10 & 0.53 & $\mathrm{~L}$ \\
\hline
\end{tabular}

Mobile phase; $10 \%$ 2-propanol/hexane (V/V) containing $0.1 \%$ TFA; Flow rate $=1 \mathrm{~mL} / \mathrm{min}$; Detector $254 \mathrm{~nm} .{ }^{a}$ Capacity factor for the first eluted enantiomer. ${ }^{b}$ Separation factor. ${ }^{c}$ Resolution factor. ${ }^{d}$ indicates the absolute configuration of the more strongly retained enantiomer. ${ }^{e} 20 \% 2$ propanol/hexane $(\mathrm{V} / \mathrm{V})$ containing $0.1 \%$ TFA ${ }^{f} 15 \%$ 2-propanol/hexane (V/V) containing $0.1 \%$ TFA.

or better enantioselectivities for the resolution of $N$-FMOC $\alpha$-amino acids than Chiralpak AD except $N$-FMOC protected glutamine and tyrosine analytes (entries 7 and 17). All investigated $N$-FMOC $\alpha$-amino acids enantiomers were base-line separated on Chiralcel OD or Chiralpak AD. For example, while $N$-FMOC glutamine, norvaline, phenylalanine and tyrosine enantiomers were partially separated on Chiralcel OD $(\mathrm{Rs}<1)$, all these enantiomers were base-line resolved on Chiralpak AD (Rs > 1.48). Therefore, Chiralcel OD and Chiralpak AD might be complementarily used for the separation of the enantiomers of $N$-FMOC $\alpha$-amino acids. The consistent elution order of the resolved $N$-FMOC $\alpha$-amino acids is not observed on Chiralcel OD and Chiralpak AD. As shown in Table 1, the elution of the (D)isomers prior to the (L)-isomers for resolution of $N$-FMOC $\alpha$-amino acids examined except five analytes is observed on 
Table 2. Enantiomer Separation of $N$-FMOC Protected $\alpha$-Amino Acids on Chiralpak AD

\begin{tabular}{clrccc}
\hline Entry & \multicolumn{1}{c}{ Analyte } & \multicolumn{1}{c}{$\mathrm{k}_{1}{ }^{a}$} & $\alpha^{b}$ & $\mathrm{Rs}^{c}$ & Retained $^{d}$ \\
\hline 1 & Alanine & 2.98 & 1.12 & 1.44 & $\mathrm{D}$ \\
2 & Aminobutyric acid & 3.23 & 1.12 & 1.37 & $\mathrm{D}$ \\
3 & Aminocaprylic acid & 2.75 & 1.12 & 1.36 & \\
4 & Asparagine & 6.65 & 1.21 & 2.10 & $\mathrm{~L}$ \\
5 & Aspartic acid & 4.70 & 1.17 & 0.94 & $\mathrm{D}$ \\
6 & Glutamic acid & 5.14 & 1.26 & 2.72 & $\mathrm{~L}$ \\
7 & Glutamine & 5.42 & 1.44 & 3.83 & $\mathrm{~L}$ \\
8 & Isoleucine & 3.71 & 1.19 & 1.99 & $\mathrm{D}$ \\
9 & Leucine & 3.02 & 1.18 & 1.83 & $\mathrm{~L}$ \\
10 & Methionine & 5.38 & 1.00 & - & - \\
11 & Norleucine & 9.44 & 1.07 & 0.94 & $\mathrm{D}$ \\
12 & Norvaline & 10.19 & 1.11 & 1.60 & $\mathrm{D}$ \\
13 & Phenylalanine & 4.59 & 1.12 & 1.48 & $\mathrm{~L}$ \\
14 & Phenylglycine & 7.12 & 1.38 & 4.24 & $\mathrm{D}$ \\
15 & Serine & 4.40 & 1.10 & 1.07 & $\mathrm{~L}$ \\
16 & Threonine & 4.89 & 1.13 & 1.50 & $\mathrm{D}$ \\
17 & Tyrosine & 23.79 & 1.29 & 3.45 & $\mathrm{~L}$ \\
18 & Valine & 3.99 & 1.12 & 1.38 & $\mathrm{D}$ \\
\hline
\end{tabular}

Mobile phase; $10 \%$ 2-propanol/hexane $(\mathrm{V} / \mathrm{V})$ containing $0.1 \%$ TFA; Flow rate $=1 \mathrm{~mL} / \mathrm{min}$; Detector $254 \mathrm{~nm}$. ${ }^{a}$ Capacity factor for the first eluted enantiomer. ${ }^{b}$ Separation factor. ${ }^{c}$ Resolution factor. ${ }^{d}$ indicates the absolute configuration of the more strongly retained enantiomer. ${ }^{e} \% 2-$ propanol/hexane $(\mathrm{V} / \mathrm{V})$ containing $0.1 \% \mathrm{TFA}$.

Chiralcel OD. The chromatographic method in this study was used for determination of the enantiomeric purity of two commercially available and one synthesized $N$-FMOC phenylglycine analytes on Chiralcel OD, as shown in Table 3. ${ }^{16,18}$ The enantiomeric impurities of $0.5-0.8 \%$ for these samples can be determined in the mobile phase of $20 \% 2$ propanol/hexane (V/V) containing $0.1 \%$ trifluoroacetic acid (TFA). Chromatograms of determination of the enantiomeric purity of commercially available $N$-FMOC D- and Lphenylglycine on Chiralcel OD are presented in Figure 1, respectively.

In summary, we demonstrated the liquid chromatographic separation of enantiomers of $N$-FMOC protected $\alpha$-amino acids on polysaccharide-derived CSPs, Chiralcel OD and Chiralpak AD. In general, the separation factors shown for resolution of $N$-FMOC $\alpha$-amino acids on Chiralcel OD are greater than those on Chiralpak AD. Since all investigated $N$-FMOC $\alpha$-amino acids enantiomers were base-line

Table 3. Determination of the enantiomeric purity of $N$-FMOC Land D-phenylglycine on Chiralcel OD

\begin{tabular}{cccc}
\hline Entry & Sample & Company & L $:$ D ratio $^{a}$ \\
\hline 1 & $N$-FMOC-D-phenylglycine & Fluka & $0.5: 99.5$ \\
2 & $N$-FMOC-L-phenylglycine & Fluka & $99.2: 0.8$ \\
3 & $N$-FMOC-D-phenylglycine & Synthesized $^{b}$ & $0.5: 99.5$ \\
\hline
\end{tabular}

Mobile phase; 20\% 2-propanol/hexane (V/V) containing 0.1\% TFA; Flow rate $=1 \mathrm{~mL} / \mathrm{min}$; Detector $254 \mathrm{~nm}$; Injection amount $25 \mu \mathrm{g}$. ${ }^{a}$ Average value of three times determined. ${ }^{b}$ Synthesized sample according to the conventional methods. ${ }^{17}$

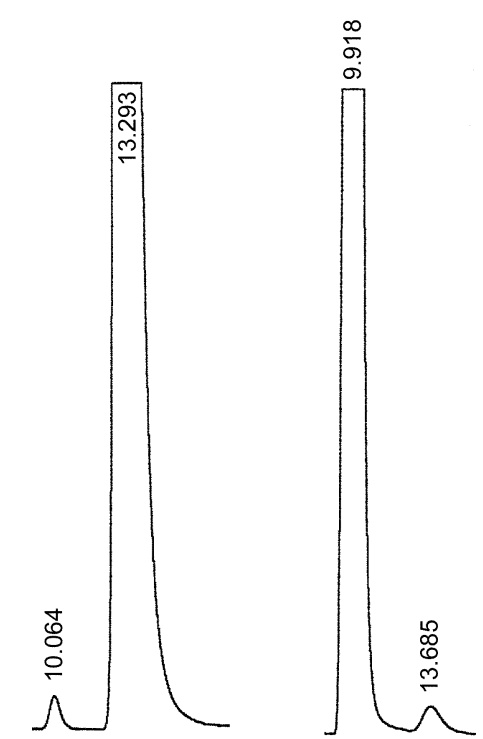

Figure 1. Chromatograms of determination of the enantiomeric purity of $N$-FMOC D-phenylglycine (the left, $\mathrm{L}: \mathrm{D}=0.5: 99.5$ ) and $N$-FMOC L-phenylglycine (the right, $\mathrm{L}: \mathrm{D}=99.2: 0.8$ ) on Chiralcel OD. See Table 3 for chromatographic conditions.

separated on Chiralcel OD or Chiralpak AD, it is expected that Chiralcel $\mathrm{OD}$ or Chiralpak $\mathrm{AD}$ will be usefully applicable to the resolution of $N$-FMOC protected $\alpha$-amino acids for determination of enantiomeric purity.

\section{Experimental Section}

Chromatographic analysis was performed at room temperature using an HPLC consisting of a Waters model 510 pump, a Rheodyne model 7125 injector with a $20 \mu \mathrm{L}$ loop, a variable wavelength UV detector Waters 490 set at $254 \mathrm{~nm}$ and an HP 3396 series II recorder. Chiralcel OD and Chiralpak AD column (250 mm L × $4.6 \mathrm{~mm}$ I.D. $)$ were purchased from Daicel Chemical Company (Tokyo, Japan). HPLC-grade hexane and 2-propanol were obtained from J. T. Baker (Phillipsburg, NJ). Trifluoroacetic acid (TFA) was obtained from Aldrich (Milwaukee, WI). The racemic (or enantiomerically pure) $N$-FMOC protected $\alpha$-amino acids were prepared according to the conventional methods. ${ }^{17} \mathrm{~N}$ FMOC D- and L-phenylglycine were obtained from Fluka company.

\section{References}

1. Greene, T. W.; Wuts, P. G. M. Protective Groups in Organic Synthesis, 3rd Ed.; John Wiley \& Sons. Inc.: New York, 1999.

2. Einarsson, S.; Folestad, S.; Josefsson, B.; Lagerkvist, S. Anal. Chem. 1986, 58, 1638.

3. Brückner, H.; Lüpke, M. J. Chromatogr. A 1996, 697, 295.

4. Burgess, K. Solid-Phase Organic Synthesis, 3rd Ed.; John Wiley \& Sons. Inc.: New York, 2000.

5. Jung, G. Combinatorial Chemistry: Synthesis, Analysis, Screening; Wiley-VCH: Tubingen, 1999.

6. Zukowski, J.; Pawlowska, M.; Armstrong, D. W. J. Chromatogr. 1992, 623, 33

7. Tang, Y.; Zukowski, J.; Armstrong, D. W. J. Chromatogr. A 1996, 
743,261

8. Ekborg-Ott, K. H.; Liu, Y.; Armstrong, D. W. Chirality 1998, 10, 434.

9. Piccinini, A.-M.; Schmid, M. G.; Pajpanova, T.; Pancheva, S.; Grueva, E.; Gubitz, G. J. Biochem. Biophys. Methods 2004, 61, 11.

10. Oi, H.; Kitahara, H.; Aoki, F.; Kisu, N. J. Chromatogr. A 1995, 689, 195.

11. Pirkle, W. H.; Lee, W. Bull. Korean Chem. Soc. 1998, 19, 1277.

12. Krawinkler, K. H.; Maier, N. M.; Sajovic, E.; Lindner, W. J. Chromatogr. A 2004, 1053, 119.
13. Knoche, B.; Milosavljev, S.; Gropper, S.; Brunner, L. A.; Powell, M. L. J. Chromatogr. B 1997, 695, 355.

14. Application guide for chiral HPLC selection, third Edition, Daicel Chemical Industries, Ltd.

15. Kim, B.-H.; Lee, W. J. Liq. Chromatogr. \& Rel. Tech. 1999, 22, 523.

16. Lee, W.; Kim, B.-H. J. High Resol. Chromatogr. 1998, 21, 189.

17. Bodansky, M.; Bodansky, A. The Practice of Peptide Synthesis; Springer: New York, 1984.

18. Perry, J. A.; Rateike, J. D.; Szczerba, T. J. J. Chromatogr. 1987, $389,57$. 